\title{
Two cases of cardiac haemangioma on cardiac magnetic resonance in adolescents
}

\author{
Zuzanna Sitarska', Marzena Barczuk-Falęcka², Łukasz A. Małek³ , Bożena Werner ${ }^{4}$, \\ Halszka Kamińska ${ }^{4}$ Michał Brzewski² \\ ${ }^{1}$ Medical University of Warsaw, Warsaw, Poland \\ ${ }^{2}$ Department of Paediatric Radiology, Medical University of Warsaw, Warsaw, Poland \\ ${ }^{3}$ Faculty of Rehabilitation, Józef Pilsudski University of Physical Education in Warsaw, Warsaw, Poland \\ ${ }^{4}$ Department of Paediatric Cardiology and General Paediatrics, Medical University of Warsaw, Warsaw, Poland
}

\section{ABSTRACT}

Cardiac haemangioma in children is a very rare benign tumour, which can lead to life-threating complications such as stroke or sudden death. There is no obvious correlation with age, sex, or family history. Symptoms depend on the location of the mass and can be non-specific. The tumour may be detected by echocardiography, but cardiac magnetic resonance (CMR) imaging allows evaluation of its size and accurate location as well the tissue characteristics. The aim of our report is to present cases of adolescent patients with cardiac haemangiomas. We present their symptoms, the diagnostic process, and possible treatment of this disease. The CMR and computed tomography (CT) images were included to present the characteristic features of cardiac haemangioma and its possible complications.

\section{KEY WORDS:}

children, cardiac magnetic resonance, cardiac haemangioma, cardiac tumour.

\section{INTRODUCTION}

We present the cases of adolescent patients with cardiac haemangioma. These cases caught our attention because cardiac haemangioma is a very rare and still not well-documented tumour. We report possible symptoms that may be present in this disease and may be encountered in clinical practice. We also include cardiac magnetic resonance (CMR) and computed tomography (CT) images of our patients to show the characteristic features of cardiac haemangioma.

\section{CASE REPORT}

A 16-year-old boy was admitted to the regional hospital due to syncope, tremors of the hands and face, chest pain, and tachycardia. A similar episode occurred two weeks earlier. On general physical examination, auscultation of his precordium revealed a silent murmur (grade 1 in Levine Grading Scale), better audible along the left sternal border. The electrocardiography, chest radiograph, and laboratory tests were unremarkable. Transthoracic echocardiography revealed a pedunculated, oval mass with heterogeneous echogenicity, situated in the apex of the right ventricle. A phase contrast computed tomography was performed to exclude a thrombus.

For further characterisation of the cardiac mass in the right ventricle, cardiac magnetic resonance imaging was performed. Steady-state free precession cine images demonstrated an $18 \times 18 \times 12 \mathrm{~mm}$ sized, round shaped, clearly delineated mass with heterogenous signal (Fig. 1, Fig. 2) - hyperintense in the central part, isointense

\section{ADDRESS FOR CORRESPONDENCE:}

Zuzanna Sitarska, Medical University of Warsaw, 61 Żwirki i Wigury St., 02-091 Warsaw, Poland, e-mail: zuzanna.pruszkowska@gmail.com 


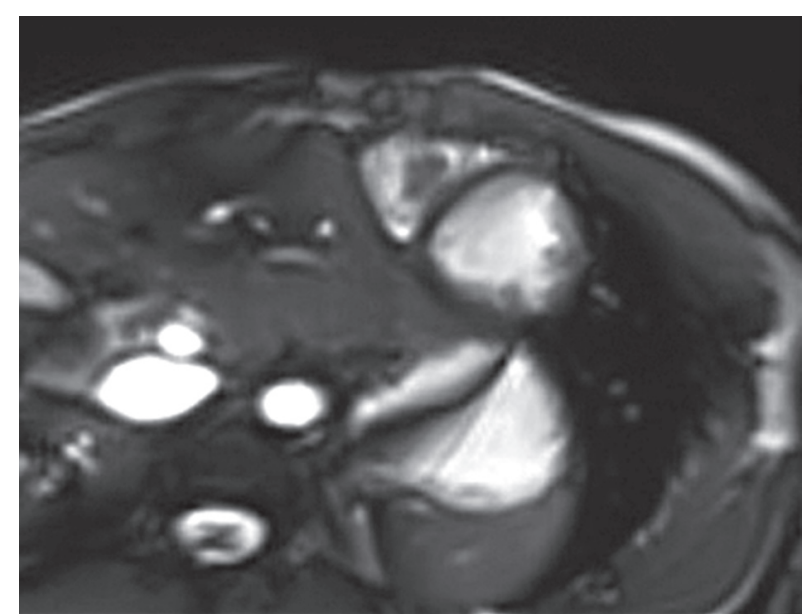

FIGURE 1. Cine image in the short-axis view at the level of the right ventricular apex

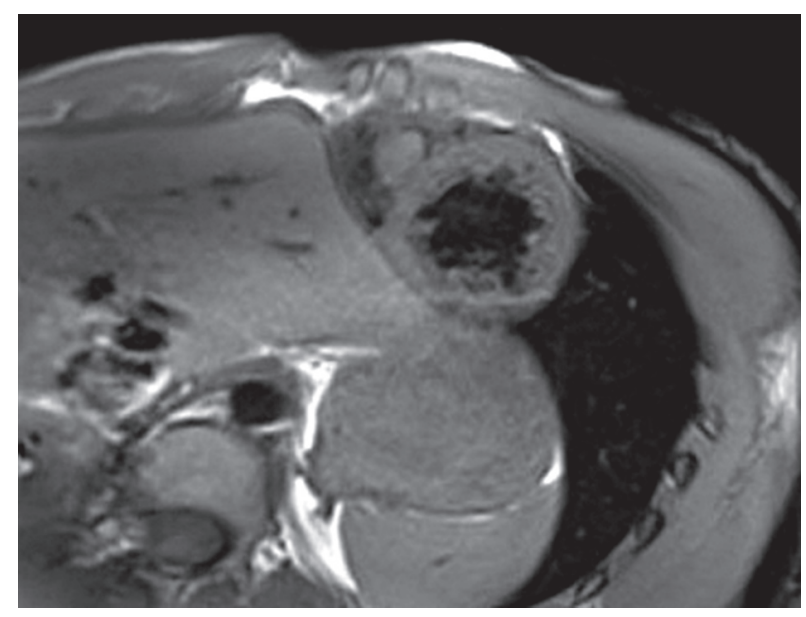

FIGURE 3. T1-weighted image with fat saturation in the short-axis view (T1-weighted image without fat saturation not shown)

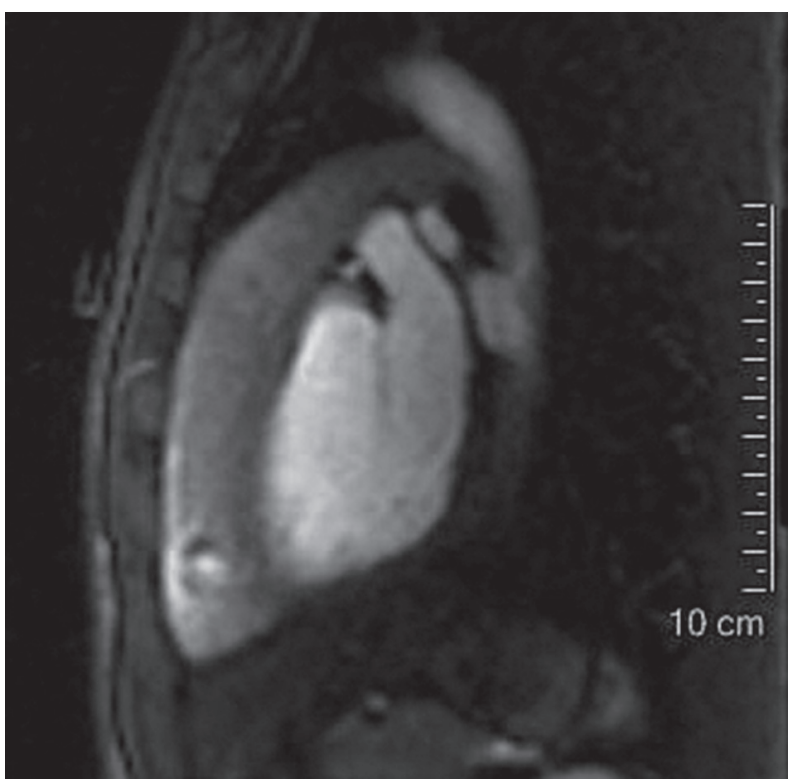

FIGURE 5. First-pass perfusion in RVOT view showing intensive centrifugal enhancement

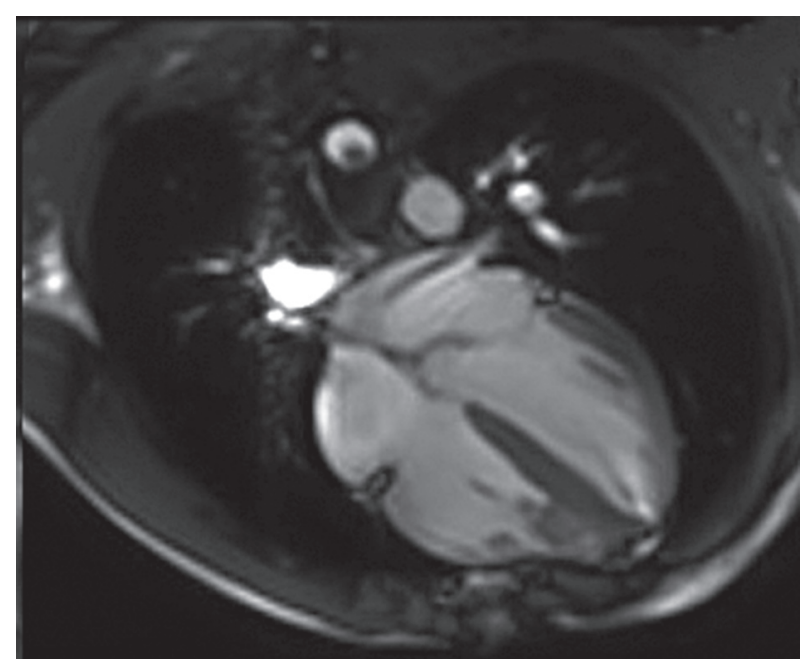

FIGURE 2. Cine image in the four-chamber view

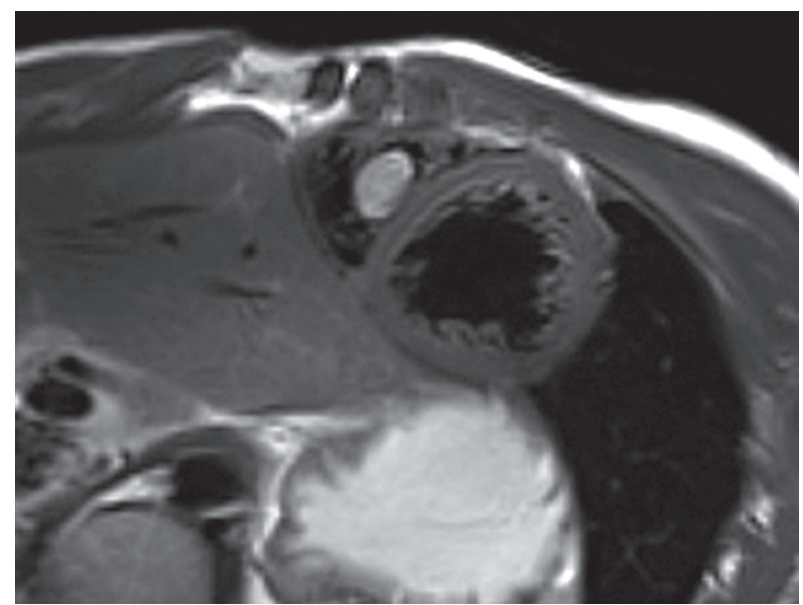

FIGURE 4. T2-weighted image in the short-axis view

peripherally. The formation was isointense and homogenous on T1-weighted images with and without fat saturation (Fig. 3) and hyperintense on T2-weighted images (Fig. 4). There was a strong first pass perfusion of gadolinium contrast in the area of the mass suggestive of good vascularisation of the structure with initially centrifugal nodular enhancement (Fig. 5) and progressive peripheral fill-in and also homogenous filling in the late phase due to delayed venous filling (Fig. 6). Diffusion-weighted imaging (DWI) showed no diffusion restriction in the mass. Right ventricular ejection fraction was preserved (54\%). The whole picture was typical for benign tumour - haemangioma. The patient was consulted by surgeons, who considered removal of the mass. The patient was discharged on his parents' request before the final consultation.

The second patient was a 17-year-old boy in whom the cardiac haemangioma was located in the basal parts of the interventricular septum. CT examination revealed the vascularisation of the tumour from the dominant right posterior descending coronary artery (Fig. 7). Because of his generally good condition, the patient has been observed for several years. CMR examination, performed 


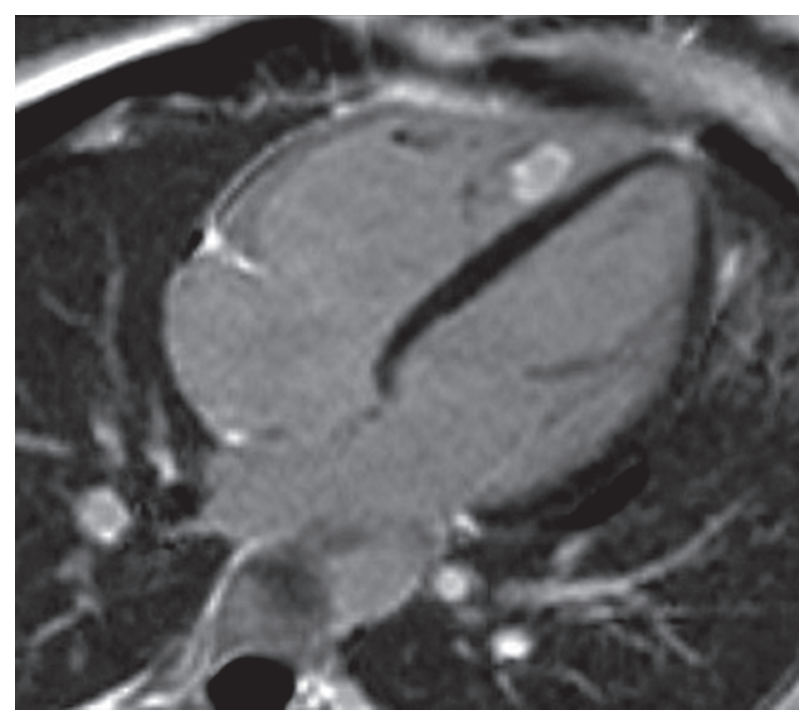

FIGURE 6. Delayed enhancement image in the four-chamber view showing homogenously enhanced mass

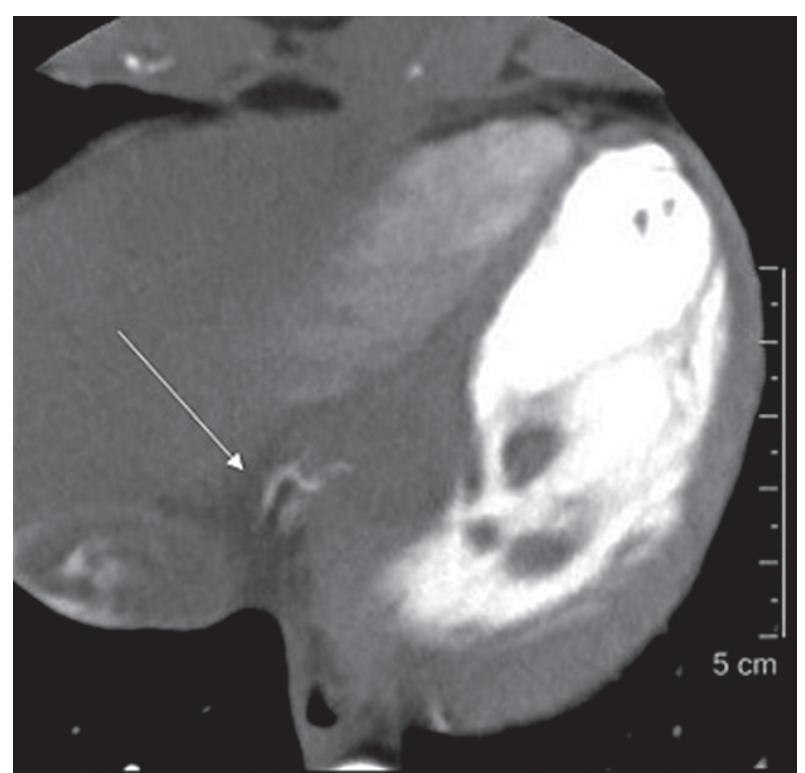

FIGURE 7. Computed tomography of the vessel supplying the tumour

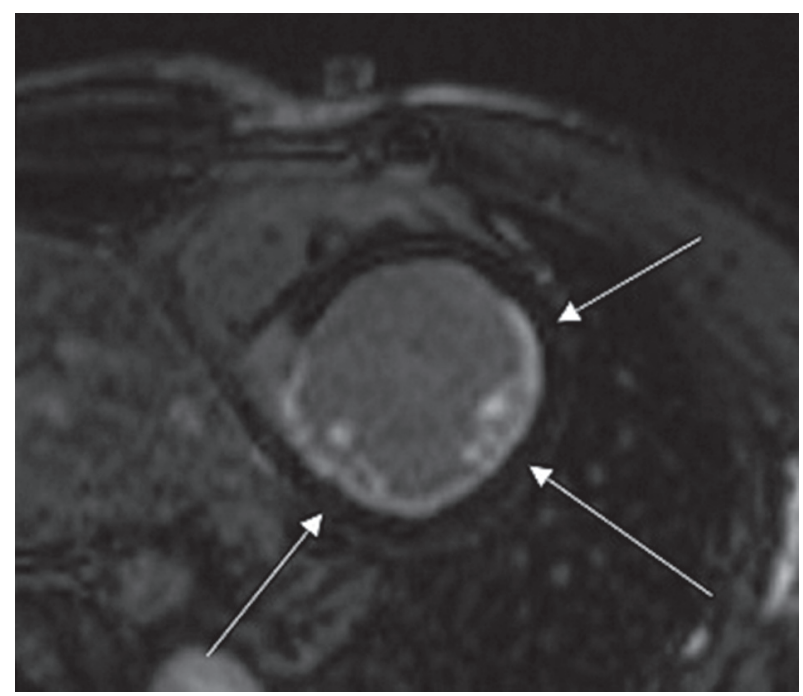

FIGURE 8. Subendocardial infarction of the inferoseptal, inferior, inferolateral, and anterolateral medial segments due to manifestation of ventricular arrhythmias and cardiac insufficiency, revealed a new finding in the form of decreased left ventricular ejection fraction to $38 \%$ due to subendocardial infarction encompassing $25 \%$ of the thickness of the inferior and lateral wall (Fig. 8). Unfortunately, the patient died suddenly awaiting further management. The cause of death was sudden cardiac death, probably due to arrhythmia or myocardial infarction.

\section{DISCUSSION}

Cardiac haemangiomas in the paediatric population are very rare and account for only $2.8 \%$ of paediatric cardiac tumours [1]. Cardiac haemangiomas are benign tumours but they can be clinically dangerous because of the risk of syncope, stroke, or even sudden death. The disease can occur at any age with no significant difference in prevalence of sex and with no obvious family history. Cardiac haemangioma can grow in any part of the heart including the endocardium, myocardium, epicardium, and pericardium $[1,2]$. Common symptoms include shortness of breath, heart palpitations, coronary insufficiency, atypical angina, or outflow tract obstruction. However, symptoms depend on the tumour's location and evolution (compression, infiltration, rupture, bleeding, embolisation, infection) [3].

Echocardiography (ECHO) is the conventional screening diagnostic modality to assess masses in the heart. Haemangioma observed in ECHO usually has mixed echogenicity with hypoechoic and cystic spaces with possible calcification and thrombosis formation [3]. The CMR can provide more accurate evaluation of the tumour size, location, and tissue characteristics.

Conservative treatment, such as radiotherapy, corticosteroid, and beta-blockers, is possible in asymptomatic patients (tumour as incidental finding), as well as in those with unresectable masses [1]. Indications for surgery in cardiac haemangiomas remain controversial. Due to the potential risk of embolism, rupture, or congestive heart failure, some suggest surgical excision [1-3]. However, because of the vascular nature of the tumour and its location, complete surgical excision may be difficult [3], which was observed in our second patient. The whole picture of his symptoms and imaging findings were suggestive of a steal phenomenon or ischaemia caused by right coronary artery compression by the tumour mass. Both situations were previously reported in adults - steal phenomenon was observed by myxoma in the left atrium as well the coronary compression by haemangioma $[4,5]$. Additionally, interseptal location was previously reported as an independent risk factor of sudden cardiac death [1].

\section{CONCLUSIONS}

In summary, we presented two cases of cardiac haemangioma in adolescents, including CMR picture and 
potential life-threatening complications in the case of interseptal location of the tumour.

\section{DISCLOSURE}

The authors declare no conflict of interest.

\section{REFERENCES}

1. Li W, Teng P, Xu H, et al. Cardiac hemangioma: a comprehensive analysis of 200 cases. Ann Thorac Surg 2015; 99: 2246-2252.

2. Delmo Walter EM, Javier MF, Sander F, et al. Primary cardiac tumors in infants and children: surgical strategy and long-term outcome. Ann Thorac Sur 2016; 102: 2062-2069.

3. Han $Y$, Chen X, Wang X, et al. Cardiac capillary hemangioma: a case report and brief review of the literature. J Clin Ultrasound 2014; 42: 53-56.

4. Stiver K, Bittenbender P, Whitson BA, et al. Left atrial myxoma causing coronary steal: an atypical cause of angina. Tex Heart Inst J 2015; 42: $270-272$.

5. Olivotti L, Botta L, Gandolfo A, et al. Coronary and atrial compression by a giant cardiac hemangioma. Cardiovasc Pathol 23, 2014: 366-368. 